\title{
Explicit methods for stiff stochastic differential equations
}

Assyr Abdulle

\begin{abstract}
Multiscale differential equations arise in the modeling of many important problems in the science and engineering. Numerical solvers for such problems have been extensively studied in the deterministic case. Here, we discuss numerical methods for (mean-square stable) stiff stochastic differential equations. Standard explicit methods, as for example the EulerMaruyama method, face severe stepsize restriction when applied to stiff problems. Fully implicit methods are usually not appropriate for stochastic problems and semi-implicit methods (implicit in the deterministic part) involve the solution of possibly large linear systems at each time-step. In this paper, we present a recent generalization of explicit stabilized methods, known as Chebyshev methods, to stochastic problems. These methods have much better (mean-square) stability properties than standard explicit methods. We discuss the construction of this new class of methods and illustrate their performance on various problems involving stochastic ordinary and partial differential equations.
\end{abstract}

\section{Introduction}

The growing need to include uncertainty in many problems in engineering and the science has triggered in recent year the development of computational methods for stochastic systems. In this paper we discuss numerical methods for stiff stochastic differential equations (SDEs). Such equations are used to model many important applications from biological and medical sciences to chemistry or financial engineering [41],[17],[34]. A main issue for practical application is the problem of stiffness. Various definitions of stiff systems for ordinary differential equations (ODEs) are proposed in the lit-

Section of Mathematics, Swiss Federal Institute of Technology (EPFL), Station 8, CH-1015, Lausanne, Switzerland. E-mail address: assyr.abdulle@epfl.ch 
erature [20] (see also [28, Chap. 9.8] for a discussion in the stochastic case). Central to the characterization of stiff systems is the presence of multiple time scales the fastest of which being stable. The usual remedy to the issue of stiffness (in the deterministic case) is to use implicit methods. This comes at the cost of solving (possibly large and badly conditioned) linear systems. For classes of problems (dissipative problems), explicit methods with extended stability domains, called Chebyshev or stabilized methods, can be efficient [25],[29],[2],[3] and have proved successful in applications (see for example [15],[22],[19],[4] to mention but a few). In this paper we review the recent extensions [7],[6],[8],[9] of Chebyshev methods to mean-square stable stochastic problems with multiple scales.

We close this introduction by mentioning that the stability concept considered in this paper, namely the mean-square stability, does not cover some classes of interesting multiscale stochastic systems. Indeed, adding noise to a deterministic stiff system (where Chebyshev or implicit methods are efficient) may lead to stochastic problems for which the aforementioned methods are not accurate. Adding for example a suitably scaled noise $\left(\sqrt{\frac{1}{\varepsilon}} d W(t)\right)$ to the fast system of the following singular perturbed problem

$$
\begin{aligned}
& d x=f(x, y) d t, x\left(t_{0}\right)=x_{0}, \\
& d y=\frac{1}{\varepsilon} g(x, y) d t, y\left(t_{0}\right)=y_{0},
\end{aligned}
$$

where $\varepsilon>0$ is a small parameter, can lead to a fast system with a non-trivial invariant measure. To capture numerically the effective slow variable, requires to correctly compute the invariant measure of the fast system. This might not be possible for implicit ${ }^{1}$ or Chebyshev methods, if one uses large stepsize for the fast process. Even though such problems are not mean-square stable, the stability properties of implicit or Chebyshev methods still allow to compute trajectories which remain bounded. But the damping of these methods may prevent the capture of the right variance of the invariant distribution (see [30], [8] for examples and details). In such a situation one should use methods relying on averaging theorems as proposed in [39] and [16].

The paper is organized as follows. In Section 2 we discuss stiff stochastic systems and review the mean-square stability concept for the exact and the numerical solution of an SDE. Next, in Section 3 we introduce the Chebyshev methods for stiff ODEs. The extension of such methods to SDEs (called the S-ROCK methods) are presented in Section 4. In Section 5, we study the stability properties of the S-ROCK methods. Numerical comparison illustrating the performance of the S-ROCK methods and comparison with several standard explicit methods for SDEs are given in Section 6 .

\footnotetext{
1 There is one exception, namely the implicit midpoint rule, which works well for (1)-(2) when the fast process is linear in $y$. This is due to the lack of damping at infinity [30].
} 


\section{Stiff stochastic systems and stability}

As an illustrative example, consider the following stochastic partial differential equation (SPDE), the heat equation with noise (see [7]):

$$
\frac{\partial u}{\partial t}(t, x)=D \frac{\partial^{2} u}{\partial x^{2}}(t, x)+\mu u(t, x) \dot{W}(t), t \in[0, T], x \in[0,1],
$$

where we choose the initial conditions $u(0, x)=1$, and mixed boundary conditions $u(t, 0)=5,\left.\frac{\partial u(t, x)}{\partial x}\right|_{x=1}=0$ and $D=1$. Here $\dot{W}(t)$ denotes a white noise in time ${ }^{2}$. To solve numerically the above system, we follow the method of lines (MOL) and discretize first the space variable

$$
d Y_{t}^{i}=\frac{Y_{t}^{i+1}-2 Y_{t}^{i}+Y_{t}^{i-1}}{h^{2}}+\mu Y_{t}^{i} d W_{t}, \quad i=1, \ldots, N,
$$

to obtain (a large) system of $N$ SDEs, where $N=\mathcal{O}(1 / h)$.

Remark 1. Notice that we used finite differences (FDs) to perform the spatial discretization. We emphasize that finite element methods (FEMs) could have been used as well. In a first step one would obtain a system $M Y^{\prime}=\ldots$, where $M$ is the mass matrix. For low order FEs a cheap procedure, called mass lumping, allows to transform $M$ into a diagonal matrix without loss of accuracy for the numerical method [38].
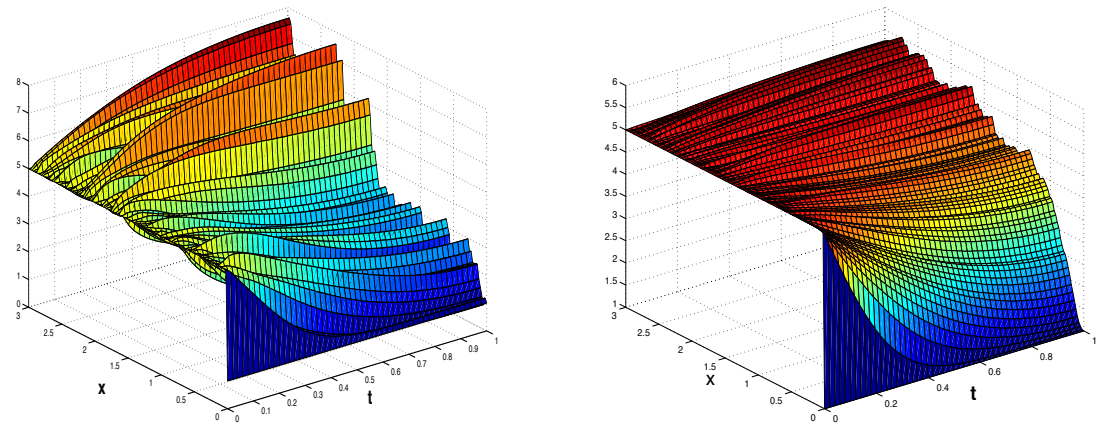

Fig. 1 One realisation of the system (4) with the Euler-Maruyama method (left figure); average over 100 realizations (right figure). Parameters values: $D=k=1, N=50, \Delta t=$ $2^{-14}, t \in[0,3]$.

We first write the system (4) in the form $d Y=(A Y+B(Y)) d t+G Y d W_{t}$, where $A$ is a tridiagonal matrix (approximation of the second order partial

${ }^{2}$ We will not discuss the precise meaning of (3), whose rigorous definition involves an integral equation [40],[14]. 
differential operator), $B(Y)$ is a vector accounting for the boundary conditions, and $G$ is a (diagonal) matrix accounting for the multiplicative noise. When then obtain after (simultaneous) diagonalization, the system of SDEs (with appropriate boundary conditions omitted here) reads

$$
d Y_{t}^{i}=\lambda_{i} Y_{t}^{i} d t+\mu Y_{t}^{i} d W_{t}, \quad i=1, \ldots, N,
$$

where $\lambda_{i} \in\left[-\mathcal{O}\left(N^{2}\right), 0\right]$ (see [7] for details). As for (3), the rigorous interpretation of (5) is an integral form involving a stochastic integral for which various "calculus" can be used, most often the Itô or the Stratonovich calculus [10]. The numerical methods described in this paper have been derived for both calculus. For the time being, we will consider Itô form. The simplest numerical scheme to solve (5) (assuming Itô form) is the Euler-Maruyama method, a generalization of the Euler scheme for ordinary differential equations (ODEs) introduced in [32]

$$
Y_{n+1}=Y_{n}+\Delta t \lambda Y_{n}+I_{n} \mu Y_{n}
$$

where $I_{n}=W\left(t_{n+1}\right)-W\left(t_{n}\right)$ are independent normal $\mathcal{N}(0, \Delta t)$ random variables.

As for ODEs, two important issues arise when deriving numerical methods for SDEs, namely the accuracy and the stability of the approximation procedure.

Accuracy. Consider

$$
d Y=f(t, Y) d t+\sum_{l=1}^{M} g_{l}(t, Y) d W_{l}(t), \quad Y(0)=Y_{0},
$$

where $Y(t)$ is a random variable with values in $\mathbb{R}^{d}, f:[0, T] \times \mathbb{R}^{d} \rightarrow \mathbb{R}^{d}$ is the drift term, $g:[0, T] \times \mathbb{R}^{d} \rightarrow \mathbb{R}^{d}$ is the diffusion term and $W_{l}(t)$ are independent Wiener processes. Assuming that $f$ and $g$ are continuous, have a linear growth and are uniform Lipschitz continuous with respect to the variable $Y$, that $Y_{0}$ has finite second order moment and is independent of the Wiener processes, one can show the existence and uniqueness of a (meansquare bounded) strong solution of (7) (see for example [33, Chapter 5.2] for details). Consider for the numerical approximation of (7) the one-step method of the form

$$
Y_{n+1}=\Phi\left(Y_{n}, \Delta t, I_{n_{1}}, \ldots, I_{n_{M}}\right),
$$

where $I_{n_{l}}=W_{l}\left(t_{n+1}\right)-W_{l}\left(t_{n}\right)$ are independent Wiener increments drawn from the normal distributions with zero mean and variance $\Delta t=t_{n+1}-t_{n}$. The numerical method (8) is said to have a strong order $\rho$, respectively weak order of $\rho$, if there exists a constant $C$ such that 
$\mathbb{E}\left(\left|Y_{n}-Y(\tau)\right|\right) \leq C(\Delta t)^{\rho}$, respectively $\left|\mathbb{E}\left(G\left(Y_{n}\right)\right)-\mathbb{E}(G(Y(\tau)))\right| \leq C(\Delta t)^{\rho}$,

for any fixed $\tau=n \Delta t \in[0, T]$ ( $\Delta t$ sufficiently small) and for all functions $G: \mathbb{R}^{d} \rightarrow \mathbb{R} 2(\rho+1)$ times continuously differentiable with partial derivatives having polynomial growth.

Remark 2. In general, for numerical methods depending only on the first Wiener increment $W_{l}\left(t_{n+1}\right)-W_{l}\left(t_{n}\right)$ the highest strong and weak order that can be obtained are $1 / 2$ and 1 , respectively. Strong order one can be obtained for 1-dimensional problems or if commutativity conditions hold for the diffusion functions $g_{l}[36],[28],[13]$.

Stability. We have to investigate for what $\Delta t$ does a numerical method $Y_{n+1}=\Phi\left(Y_{n}, \Delta t, I_{n_{1}}, \ldots, I_{n_{M}}\right)$ applied to $(7)$ share the stability properties of the exact solution $Y_{t}$. Widely used measures of stability for SDEs are meansquare stability, which measures the stability of moments, and asymptotic stability (in the large), which measures the overall behavior of sample functions [21]. We will focus here on mean-square stability. For linear autonomous system of SDEs, this concept of stability is stronger than asymptotic stability (see [10, Chap. 11]) or [21]). Consider the $\operatorname{SDE}(7)$ with $f(t, 0)=g_{l}(t, 0)=0$ and with a nonrandom initial value $Y_{0}$. The steady solution $Y=0$ of $(7)$ is said to be mean-square stable if there exists $\delta_{0}$ such that

$$
\lim _{t \rightarrow \infty} \mathbb{E}\left(|Y(t)|^{2}\right)=0, \text { for all }\left|Y_{0}\right|<\delta_{0} .
$$

In order to analyze the stability of numerical methods one has to restrict the class of problems considered. Inspired by (5) and following [37],[23] we consider the scalar linear test equation

$$
d Y=\lambda Y d t+\mu Y d W(t), \quad Y(0)=Y_{0},
$$

where $\lambda, \mu \in \mathbb{C}$. For $\mu=0$ one recovers the Dahlquist test equation, which is instrumental in developing the linear $A$-stability theory for ODEs [20, Chap. $4.2,4.3]$.

Remark 3. We note that for SDEs, it is at first not clear to which extend the study of a scalar linear test problem is relevant to systems of linear equations or fully nonlinear equations. Recent work, however, suggest that stability analysis for the scalar test equation is relevant for more general systems [11].

The test equation (11) can be solved analytically and the solution reads

$$
Y(t)=Y_{0} e^{\left(\left(\lambda-\frac{\mu^{2}}{2}\right) t+\mu W(t)\right)}\left(\text { Itô) }, Y(t)=Y_{0} e^{(\lambda t+\mu W(t))}\right. \text { (Stratonovich), }
$$

and we have for the mean-square stability 


$$
\lim _{t \rightarrow \infty} \mathbb{E}\left(|Y(t)|^{2}\right)=0 \Longleftrightarrow\left\{\begin{array}{l}
\left\{(\lambda, \mu) \in \mathbb{C}^{2} ; \Re \lambda+\frac{1}{2}|\mu|^{2}<0\right\} \text { (Itô), } \\
\left\{(\lambda, \mu) \in \mathbb{C}^{2} ; \Re \lambda+(\Re \mu)^{2}<0\right\} \text { (Stratonovich). }
\end{array}\right.
$$

If we apply the Euler-Maruyama method (6) to (11) we obtain

$$
\mathbb{E}\left(\left|Y_{n+1}\right|^{2}\right)=\left(|1+p|^{2}+q^{2}\right) \mathbb{E}\left(\left|Y_{n}\right|^{2}\right),
$$

where $p=\Delta t \lambda, q=\sqrt{\Delta t} \mu$ and thus, the method is mean-square stable if and only if $|1+p|^{2}+q^{2}<1$. More generally, if we apply the numerical scheme (8) to the test problem (11), square the result and take the expectation, we obtain

$$
\mathbb{E}\left(\left|Y_{n+1}\right|^{2}\right)=R(p, q) \mathbb{E}\left(\left|Y_{n}\right|^{2}\right),
$$

where $p=\Delta t \lambda, q=\sqrt{\Delta t} \mu$ and where $R(p, q)$ is a function in $\Re(p), \Im(p)$, $\Re(q), \Im(q)$ (a polynomial in these variables if the method is explicit). We say that a numerical method is mean-square stable for the test problem (11) if and only if

$$
\lim _{n \rightarrow \infty} \mathbb{E}\left(\left|Y_{n}\right|^{2}\right)=0 \Longleftrightarrow(\Delta t \lambda, \sqrt{\Delta t} \mu) \in \mathcal{S}:=\{p, q \in \mathbb{C} ; R(p, q)<1\} .
$$

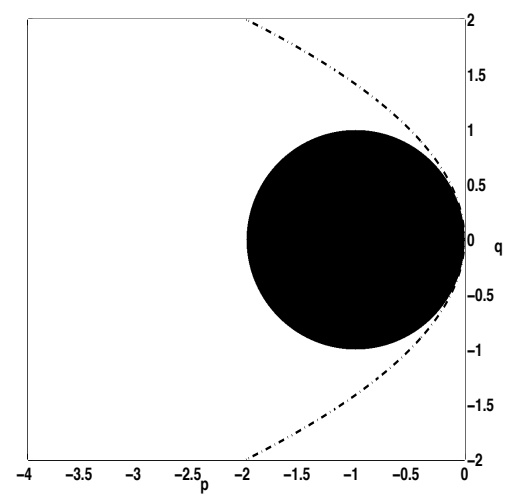

Fig. 2 Stability domain of the Euler-Maruyama method (black disk) for $\lambda, \mu \in \mathbb{R}$. The dashed curve represent the boundary of the exact stability domain (the left part of the curve lies in the stability domain).

In order to be able to visualize the stability region, we restrict ourself to the case $\lambda, \mu \in \mathbb{R}$. We see in Figure 2 that the stability domain of the Euler-Maruyama method is a disk of radius 1 centered at $p=-1$, while the stability domain of the exact test problem is the unbounded region on the left of the dashed curve. The Euler-Maruyama has thus a restricted stability region. For the problem (3) (see also (5)) this explicit method will thus face a severe time step restriction due to stability constraint (see Figure 7 in Section 
6). One could use semi-implicit methods (implicit method in the drift term) to obtain method with much better stability properties. This comes however with the cost of solving nonlinear equations at each stepsize. This can be numerically expensive for large systems (see e.g. (4)), specially if one needs to simulate many realizations. We will explain in the next section how meansquare stability can be improved without giving up the explicitness of the numerical method.

\section{Chebyshev methods}

Chebyshev methods are a class of explicit one-step methods with extended stability domains along the negative real axis. The basic idea for such methods goes back to the 1960s with Saul'ev, Franklin and Guillou and Lago (see [20, Sect. IV.2] and the references therein). It can be summarized as follows: consider a sequence of forward Euler methods $\Psi_{h_{1}}, \ldots, \Psi_{h_{m}}$ with a corresponding sequence of timesteps $h_{1}, \ldots, h_{m}$ and define a one-step method as the composition $\Psi_{\Delta t}=\left(\Psi_{h_{m}} \circ \ldots \circ \Psi_{h_{1}}\right)\left(y_{0}\right)$ with stepsize $\Delta t=h_{1}+\ldots+h_{m}$. Next, given $m$, optimize the sequence $\left\{h_{i}\right\}_{i=1}^{m}$, so that

$$
\left|R_{m}(x)\right|=\left|\prod_{i=1}^{m}\left(1+\frac{h_{i} x}{\Delta t}\right)\right| \leq 1 \text { for } x \in\left[-l_{m}, 0\right],
$$

with $l_{m}>0$ as large as possible. The resulting numerical method will thus be a $m$-stage method. The solution of the above optimization problem is given by shifted Chebyshev polynomials

$$
R_{m}(x)=T_{m}\left(1+x / m^{2}\right)=1+x+a_{2} x^{2}+\ldots+a_{m} x^{m},
$$

where $\left\{T_{j}(x)\right\}_{j \geq 0}$ are the Chebyshev polynomials given recursively by

$$
T_{0}(x)=1, T_{1}(x)=x, \quad T_{j}(x)=2 x T_{j-1}(x)-T_{j-2}(x), j \geq 2 .
$$

We see that the optimal sequence of $\left\{h_{i}\right\}_{i=1}^{m}$ is given by $h_{i}=\left(-1 / x_{i}\right) \Delta t$, where $x_{i}$ are the zeros of $R_{m}(x)$ and the maximal stability domain on the negative real axis increases quadratically with the number of stages $m$ and is given by $l_{m}=2 m^{2}$. The property $R_{m}(z)=1+x+\mathcal{O}\left(x^{2}\right)$ ensure the first order convergence of the numerical method. Besides the stability of the "super stepsize" $\Delta t$, one has also to care about the internal stability (accumulation of errors within one step) of the method as $m$ can be large. This can be achieved either by a proper ordering of the Euler steps $h_{i}$ [29] or by exploiting the three-term recurrence relation of the orthogonal polynomials [25]. Following the second strategy we consider a $m$-stage numerical method given by 


$$
\begin{aligned}
& k_{0}:=y_{0} \\
& k_{1}:=y_{0}+\frac{\Delta t}{m^{2}} f\left(k_{0}\right) \\
& k_{j}:=\frac{2 \Delta t}{m^{2}} f\left(k_{j-1}\right)+2 k_{j-1}-k_{j-2}, 2 \leq j \leq m \\
& y_{1}:=k_{m}
\end{aligned}
$$

Applied to the test problem $y^{\prime}=\lambda y$, this method gives for the internal stages

$$
k_{j}=T_{j}\left(1+\Delta t \lambda / m^{2}\right) y_{0}, j=0, \ldots, m,
$$

and produces after one step $y_{1}=R_{m}(\Delta t \lambda) y_{0}$, where $R_{m}(x)=T_{m}\left(1+x / m^{2}\right)$, is the shifted Chebyshev polynomial of degree $m(x=\Delta t \lambda)$.

These methods have been originally developed for deterministic problems with eigenvalues along the negative real axis. A typical (deterministic) stability domain $\mathcal{S}_{m}$ of a Chebyshev method is sketched in Figure 3 (left figure), where

$$
\mathcal{S}_{m}:=\left\{z \in \mathbb{C} ;\left|R_{m}(z)\right|<1\right\} .
$$

Recall that for the linear stability of deterministic ODE solvers, one considers (11) with $\mu=0$ [20, Chap. 4.2,4.3]. It can be seen in Figure 3 that the

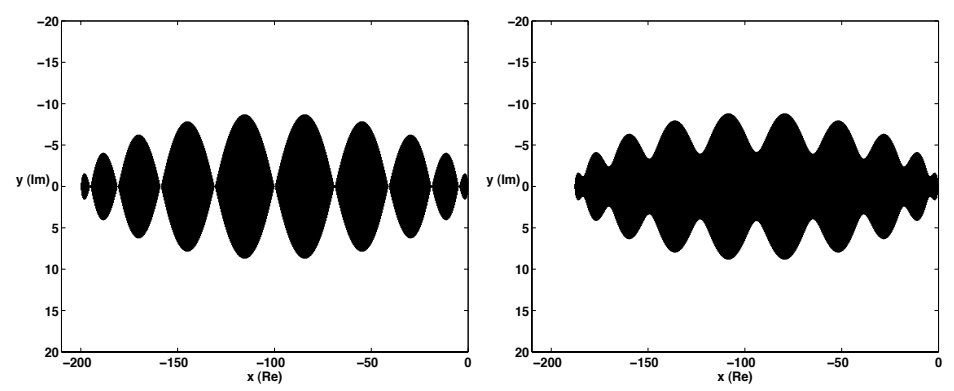

Fig. 3 Stability domain of first order Chebyshev method (degree $m=10$ ) with variable damping $\eta=0$ (left figure), $\eta=0.1$ (right figure).

boundary of the stability domain along the negative real axis is 200 , for $m=$ 10. However, there are regions in $[0,200]$, precisely when $T\left(1+x / m^{2}\right)=1$, with no stability in the direction of the imaginary axis.

To overcome the aforementioned issue, it has been suggested by Guillou and Lago [18] to replace the requirement $\left|R_{m}(x)\right| \leq 1$ in $\left[-l_{m}, 0\right]$ by $\left|R_{m}(x)\right| \leq \eta<1$ in $\left[-l_{m}^{\eta},-\epsilon\right]$, where $\epsilon$ is a small positive number. The number $\eta$ is called the damping parameter or sometimes just the "damping". This can done for the polynomials $T_{m}\left(1+x / m^{2}\right)$ by a division with $T_{m}\left(\omega_{0}\right)>1$, where $\omega_{0}=1+\eta / m^{2}$. To obtain the right order of accuracy with this modified stability function, one does a change of variables and obtains $R_{m, \eta}(x)=\frac{T_{m}\left(\omega_{0}+\omega_{1} x\right)}{T_{m}\left(\omega_{0}\right)}$, where $\omega_{1}=\frac{T_{m}\left(\omega_{0}\right)}{T_{m}^{\prime}\left(\omega_{0}\right)}$ (see [20, Section IV.2]). By increasing the parameter $\eta$ the strip around the negative real axis included 
in the stability domain can be enlarged as can be seen in Figure 3 (notice that this reduces the value of $l_{m}$ as $l_{m}^{\eta}<l_{m}$ for $\eta>0$ ). The formula (18) can be modified appropriately to incorporate damping.

Higher order quasi-optimal Chebyshev methods: the ROCK methods. Higher order methods, called ROCK, for orthogonal Runge-Kutta Chebyshev methods, based on orthogonal polynomials have been developed in [2],[3]. The stability functions are given by polynomials $R_{m}(x)=$ $1+x+\ldots+x^{p} / p !+\mathcal{O}\left(x^{p+1}\right)$ of order $p$ (i.e., $\left.R_{m}(x)-e^{x}=\mathcal{O}\left(x^{p+1}\right)\right)$ and degree $m$ with quasi optimal stability domains along the negative real axis. These polynomials can be decomposed as [1]

$$
R_{m}(x)=w_{p}(x) P_{m-p}(x),
$$

where $P_{m-p}(x)$ is a member of a family of polynomials $\left\{P_{j}(x)\right\}_{j \geq 0}$ orthogonal with respect to the weight function $\frac{w_{p}(x)^{2}}{\sqrt{1-x^{2}}}\left(w_{p}(x)\right.$ is a polynomial of degree $p$ with only complex zeros when $p$ is even and with only one real zero when $p$ is odd). ${ }^{3}$ The idea for the construction of a numerical method is then as follows: the 3 -term recurrence relation of the orthogonal polynomials $\left\{P_{j}(x)\right\}_{j \geq 0}$

$$
P_{j}(x)=\left(\alpha_{j} x-\beta_{j}\right) P_{j-1}(x)-\gamma_{j} P_{j-2}(x),
$$

is used to define the internal stages of the method

$$
K_{j}=\Delta t \alpha_{j} f\left(K_{j-1}\right)-\beta_{j} K_{j-1}-\gamma_{j} K_{j-2}, j=2, \ldots, m-p .
$$

This ensures the good stability properties of the method. A $p$-stage finishing procedure with the polynomial $w_{p}(z)$ as underlying stability function ensures the right order of accuracy of the method.

Gain in efficiency. Assume that $\Delta t$ is the stepsize corresponding to the desired accuracy to solve an initial value problem $y^{\prime}=f(t, y)$ in the interval $[0, T]$. Let $\rho$ be the spectral radius of the Jacobian $\partial_{y} f$. A standard explicit method, as the Euler method, must satisfy $\delta t=C / \rho$ (for stability) and thus needs $\Delta t \rho / C$ function evaluations in each interval $\Delta t$. For a Chebyshev method, we can select a stage number $m=\sqrt{\Delta t \rho / C}$. As the number of function evaluations is equal to the stage number of the Chebyshev method ${ }^{4}$ only the square root of the function evaluations needed for standard explicit method are required for each stepsize (notice that the constant $C$ can be different for the two methods but is in both cases of moderate size).

\footnotetext{
3 The ROCK methods have been developed for $p$ even $(p=2,4)$. They could be obtained for $p$ odd provided a proper treatment of the real zero of $w_{p}(x)$.

${ }^{4}$ Strictly speaking this is true for first order Chebyshev methods. For higher order methods, as the ROCK methods, the number of function evaluations is not equal but still close to the stage number $m$ (see [2],[3]).
} 


\section{The S-ROCK methods}

We now present the Stratonovich and the Itô stochastic ROCK (S-ROCK) methods derived in [7],[6],[8]. When modeling physical systems with SDEs, the question of the choice of the stochastic integral arises. SDEs with Stratonovich integrals are stable with respect to changes in random terms and are often used for systems where the noise is "added" as fluctuation of a deterministic system. SDEs with Itô integrals are preferred for systems with internal noise where the fluctuation is due to the systems itself as for example in chemical reactions due to the property of "not looking into the future" of the Itô integral (i.e., the martingale property) [28],[33]. Of course, there are conversion rules from one calculus to the other. However, these rules involve the differentiation of the diffusion term which can be cumbersome and costly. It is thus preferable to derive genuine formulas for both calculus. Furthermore, it is sometimes desirable to have stabilized explicit methods for discrete noise. This has been considered in [9], where the $\tau$-ROCK methods have been developed and we briefly comment on these methods as well in what follows.

\subsection{Construction of the S-ROCK methods}

Inspired by the ROCK methods, we consider methods based on

- deterministic Chebyshev-like internal stages to ensure good stability properties (stages $1,2, \ldots, m-1$ ),

- a finishing stochastic procedure to incorporate the random process and obtain the desired stochastic convergence properties.

As for deterministic methods, the use of damping plays a crucial role and allows to enlarge the width of the stability domains in the direction of the "stochastic axis" (e.g, the $q$ axis in Figure 2). This is discussed in Section 5. Deterministic Chebyshev stages. Define the $m-1$ stages of the S-ROCK method by

$$
\begin{gathered}
K_{0}=Y_{n}, \\
K_{1}=Y_{n}+\Delta t \frac{\omega_{1}}{\omega_{0}} f\left(K_{0}\right), \\
K_{j}=2 \Delta t \omega_{1} \frac{T_{j-1}\left(\omega_{0}\right)}{T_{j}\left(\omega_{0}\right)} f\left(K_{j-1}\right)+2 \omega_{0} \frac{T_{j-1}\left(\omega_{0}\right)}{T_{j}\left(\omega_{0}\right)} K_{j-1}-\frac{T_{j-2}\left(\omega_{0}\right)}{T_{j}\left(\omega_{0}\right)} K_{j-2},
\end{gathered}
$$

for $j=2, \ldots, m-1$, where $\omega_{0}=1+\frac{\eta}{m^{2}}, \omega_{1}=\frac{T_{m}\left(\omega_{0}\right)}{T_{m}^{\prime}\left(\omega_{0}\right)}$. Recall that $\eta$ is the damping parameter which will be optimized (see Section 5 ).

Stochastic stages. We have now to incorporate the noise in an appropriate way. While the deterministic stages are the same for the various S-ROCK 
methods, the finishing procedure will be different to take into account the various stochastic calculus of the underlying SDE and the desired accuracy of the methods.

Itô S-ROCK methods (multi-dimensional SDEs). We define the finishing procedure as

$$
\begin{aligned}
K_{m} & =2 \Delta t \omega_{1} \frac{T_{m-1}\left(\omega_{0}\right)}{T_{m}\left(\omega_{0}\right)} f\left(K_{m-1}\right)+2 \omega_{0} \frac{T_{m-1}\left(\omega_{0}\right)}{T_{m}\left(\omega_{0}\right)} K_{m-1}-\frac{T_{m-2}\left(\omega_{0}\right)}{T_{m}\left(\omega_{0}\right)} K_{m-2} \\
& +\sum_{l=1}^{M} I_{n_{l}} g_{l}\left(K_{m-1}\right) \\
Y_{n+1} & =K_{m} .
\end{aligned}
$$

Itô S-ROCK methods (commutative noise ${ }^{5}$ or one dimensional Wiener process). In that special case, one can improve the strong convergence of the method by considering the finishing procedure

$$
\begin{aligned}
K_{m-1}^{*} & =K_{m-1}+\sum_{r=1}^{M} g_{r}\left(K_{m-1}\right) I_{n_{r}} \\
K_{m-1}^{* *, l} & =K_{m-1}+\sqrt{\Delta t} g_{l}\left(K_{m-1}\right), \quad l=1,2, \ldots, M \\
K_{m} & =2 \Delta t \omega_{1} \frac{T_{m-1}\left(\omega_{0}\right)}{T_{m}\left(\omega_{0}\right)} f\left(K_{m-1}\right)+2 \omega_{0} \frac{T_{m-1}\left(\omega_{0}\right)}{T_{m}\left(\omega_{0}\right)} K_{m-1}-\frac{T_{m-2}\left(\omega_{0}\right)}{T_{m}\left(\omega_{0}\right)} K_{m-2} \\
& +\sum_{l=1}^{M} I_{n_{l}} g_{l}\left(K_{m-1}\right)+\frac{1}{2} \sum_{l=1}^{M} I_{n_{l}}\left(g_{l}\left(K_{m-1}^{*}\right)-g_{l}\left(K_{m-1}\right)\right) \\
& -\frac{1}{2} \sum_{l=1}^{M} \sqrt{\Delta t}\left(g_{l}\left(K_{m-1}^{* *, l}\right)-g_{l}\left(K_{m-1}\right)\right) \\
Y_{n+1} & =K_{m} .
\end{aligned}
$$

Remark 4. For $M=1$ the above formula can be further simplified and written as

$$
\begin{aligned}
K_{m-1}^{*} & =K_{m-1}+\sqrt{\Delta t} g\left(K_{m-1}\right) \\
K_{m} & =2 \Delta t \omega_{1} \frac{T_{m-1}\left(\omega_{0}\right)}{T_{m}\left(\omega_{0}\right)} f\left(K_{m-1}\right)+2 \omega_{0} \frac{T_{m-1}\left(\omega_{0}\right)}{T_{m}\left(\omega_{0}\right)} K_{m-1}-\frac{T_{m-2}\left(\omega_{0}\right)}{T_{m}\left(\omega_{0}\right)} K_{m-2} \\
& +I_{n} g\left(K_{m-1}\right)+\frac{I_{n}^{2}-\Delta t}{2 \sqrt{\Delta t}}\left(g\left(K_{m-1}^{*}\right)-g\left(K_{m-1}\right)\right) \\
Y_{n+1} & =K_{m} .
\end{aligned}
$$

${ }^{5}$ Consider $L^{l}=\sum_{k=1}^{d} g_{l}^{k} \frac{\partial}{\partial y^{k}}, l=1,2, \ldots, M$. Commutative noise means that the condition $L^{l} g_{r}^{k}=L^{r} g_{l}^{k} \forall l, r=1, \ldots, M ; k=1, \ldots, d$ holds for the diffusion functions [28]. 
Stratonovich S-ROCK methods (multi-dimensional SDEs). We define the finishing procedure as

$$
\begin{aligned}
K_{m-1}^{*} & =K_{m-1}+\frac{T_{m}\left(\omega_{0}\right)}{2 \omega_{0} T_{m-1}\left(\omega_{0}\right)} \sum_{l=1}^{M} I_{n_{l}} g_{l}\left(K_{m-2}\right) \\
K_{m} & =2 \Delta t \omega_{1} \frac{T_{m-1}\left(\omega_{0}\right)}{T_{m}\left(\omega_{0}\right)} f\left(K_{m-1}\right)+2 \omega_{0} \frac{T_{m-1}\left(\omega_{0}\right)}{T_{m}\left(\omega_{0}\right)} K_{m-1}-\frac{T_{m-2}\left(\omega_{0}\right)}{T_{m}\left(\omega_{0}\right)} K_{m-2} \\
& +\frac{\omega_{0} T_{m-1}\left(\omega_{0}\right)}{T_{m}\left(\omega_{0}\right)} \sum_{l=1}^{M} I_{n_{l}}\left(g_{l}\left(K_{m-1}\right)-g_{l}\left(K_{m-2}\right)\right) \\
Y_{n+1} & =K_{m} .
\end{aligned}
$$

Notice that this method has order one when solving SDEs with commutative noise or with only one Wiener process [6].

S-ROCK methods for discrete noise. The procedure explained above can be generalized to stochastic problems with other types of noise. In [9], the approximation of SDE for chemical kinetic systems has been considered. The SDE is of the form ${ }^{6}$

$$
d Y_{t}=\sum_{j=1}^{M} \nu_{j} \mathcal{P}\left(a_{j}\left(Y_{t^{-}}\right) d t\right),
$$

where $Y_{t}$ is a $N$-dimensional state vector (corresponding to the $N$ species of the reaction) with components in $\mathbb{N}, \nu_{j}$ is a state-change vector, $a_{j}$ is a propensity function (the number of possible combination of reactant molecules involved in the $j$-th reaction, times a stochastic reaction rate constant) and $\mathcal{P}\left(a_{j}\left(Y_{t^{-}}\right) d t\right)$ is a state-dependent Poisson noise. We now make the decomposition

$$
\begin{aligned}
d Y_{t} & =\sum_{j=1}^{M} \nu_{j} a_{j}\left(Y_{t-}\right) d t+\sum_{j=1}^{M} \nu_{j}\left(\mathcal{P}\left(a_{j}\left(Y_{t-}\right) d t\right)-a_{j}\left(Y_{t-}\right) d t\right) \\
& =f\left(Y_{t-}\right) d t+d Q_{t}
\end{aligned}
$$

where $f$ and $Q$ are called the drift part and jump part, respectively (see [31]). This form is similar with SDEs driven by Wiener processes, except for the different noise. Similarly as for the Itô or the Stratonovich S-ROCK methods, the $m-1$ deterministic Chebyshev stages can be applied to the drift part of (23), and the noise term can be incorporated in the finishing procedure in an appropriate way to solve (23) (we refer to [9] for details).

6 See [31] for a rigorous description of the problem. 


\subsection{Accuracy of the S-ROCK methods.}

Before considering the stability properties of our methods (the main motivation to consider the formulas introduced in Section 4.1) we briefly discuss their accuracy. As mentioned in Section 1, by considering numerical methods depending only on the first Wiener increment, strong accuracy higher than $\rho=1 / 2$ or weak accuracy higher than $\rho=1$ cannot be obtained. Only in the special case of commutative, diagonal or one dimensional noise, strong order $\rho=1$ is possible. The theorems below show that the S-ROCK methods enjoy the highest possible accuracy for numerical methods involving only the first Wiener increment.

Theorem 1 ([6],[7],[8]). For $m \geq 2$, the methods (19) (Itô) and (22) (Stratonovich) applied to (7) (with $\bar{f}$ and $g_{l}$ sufficiently smooth) satisfy

$$
\mathbb{E}\left(\left|Y_{N}-Y(\tau)\right|\right) \leq C(\Delta t)^{1 / 2}, \quad \mid \mathbb{E}\left(G\left(Y_{N}\right)\right)-\mathbb{E}(G(Y(\tau)) \mid \leq C \Delta t
$$

for any fixed $\tau=N \Delta t \in[0, T]$ and $\Delta t$ sufficiently small and for all functions $G: \mathbb{R}^{d} \rightarrow \mathbb{R}, \quad 4$ times continuously differentiable and for which all partial derivatives have polynomial growth.

Theorem 2 ([6],[7],[8]). Assume that (7) (with $f$ and $g_{l}$ sufficiently smooth) has commutative noise or that $M=1$. Then, for $m \geq 2$, the methods $(20),(21)$ (Itô) and (22) (Stratonovich) applied to (7) (with $f$ and $g_{l}$ sufficiently smooth) satisfy

$$
\mathbb{E}\left(\left|Y_{N}-Y(\tau)\right|\right) \leq C \Delta t
$$

for any fixed $\tau=N \Delta t \in[0, T]$ and $\Delta t$ sufficiently small.

For the proofs of these theorems we refer to [6],[7] (Stratonovich S-ROCK methods) and [8] (Itô S-ROCK methods).

\section{Extended mean-square stability and damping}

We study here the mean-square stability property of the S-ROCK methods. By applying any of the methods (19),(20),(21) or (22) to the scalar test problem (11), squaring the results and taking the expectation we obtain the mean-square stability function (see (15))

$$
R_{m}(p, q)=\frac{T_{m}^{2}\left(\omega_{0}+\omega_{1} p\right)}{T_{m}^{2}\left(\omega_{0}\right)}+Q_{m-1, r}(p, q),
$$

where $Q_{m-1, r}(p, q)$ is a polynomial of degree $2(m-1)$ in $p$ and of degree $2 r$ in $q$. The precise form of $Q_{m-1, r}(p, q)$ depends on the specific numerical 
method considered. Define $\Theta_{j}=\frac{T_{j}\left(\omega_{0}+\omega_{1} p\right)}{T_{j}\left(\omega_{0}\right)}$. For the method (19) we have $r=1$ and

$$
Q_{m-1,1}(p, q)=q^{2} \Theta_{m-1} .
$$

For the method (21) $r=2$ and

$$
Q_{m-1,2}(p, q)=q^{2} \Theta_{m-1}+\frac{q^{4}}{2} \Theta_{m-1}
$$

Finally, $r=2$ for the method (22) and

$$
\begin{aligned}
Q_{m-1,2}(p, q) & =q^{2}\left(\Theta_{m} \Theta_{m-2}+\left(\Theta_{m-2}\left(\frac{\omega_{1}}{\omega_{0}} p+1\right)\right.\right. \\
& \left.\left.+\omega_{0} \frac{T_{m-1}\left(\omega_{0}\right)}{T_{m}\left(\omega_{0}\right)}\left(\Theta_{m-1}-\Theta_{m-2}\right)\right)^{2}\right)+\frac{3}{4} q^{4} \Theta_{m-2}^{2}
\end{aligned}
$$

In Figure 4, we plot the mean-square stability domains for the method (19)
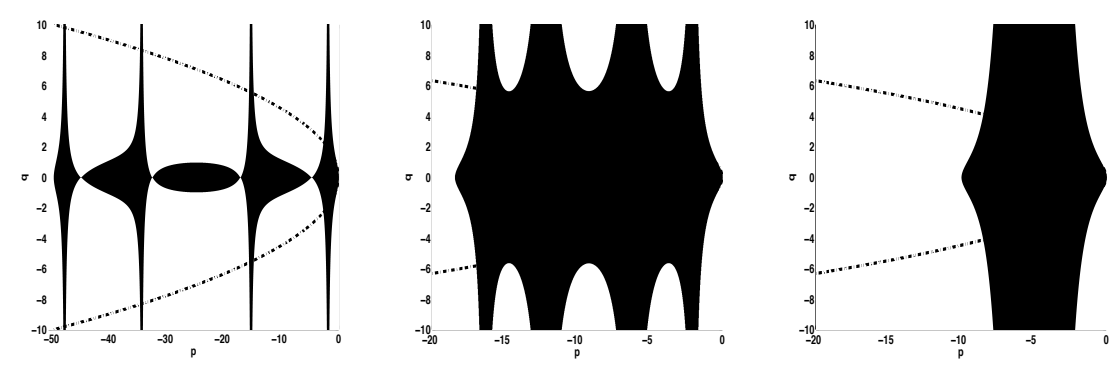

Fig. 4 Mean-square stability regions for the method (19) with various values of damping $(m=5)$. Left figure (no damping, $\eta=0$ ), middle figure (optimal damping, $\eta=4.7$ ), right figure (infinite damping).

with various values of damping for $m=5$. We observe that without damping, the stability along the $p$ axis (the "deterministic axis") is optimal (i.e., $2 \cdot 5^{2}$ ). But there are points (close to the $p$ axis) with no stability in the direction of the $q$ axis (see Figure 4 , (left)). As $Q_{m-1, r}(p, 0)=$ these points are exactly the points where $T_{m}^{2}\left(1+p / m^{2}\right)=1$. For infinite (or very large) damping the mean-square stability domain covers a portion of the stability domain of the test equation (11), but the stability domain along the $p$ axis becomes linear in $m$ (i.e., $2 \cdot 5$, see Figure 4 (right)). The mean-square stability domain for what will be called the optimal damping value covers a "large" portion of the stability domain of the test equation (see Figure 4, (middle)). In order to quantify these observations we define a "portion" of the stability domain (13) by 


$$
\mathcal{S}_{\mathrm{SDE}, s}=\{(p, q) \in[-s, 0] \times \mathbb{R} ;|q| \leq \sqrt{-p}\} \text { (Stratonovich) }
$$

or

$$
\mathcal{S}_{\mathrm{SDE}, s}=\{(p, q) \in[-s, 0] \times \mathbb{R} ;|q| \leq \sqrt{-2 p}\} \text { (Itô) },
$$

where $s>0$. We then consider two parameters $l$ and $d$ related to a numerical stability domain $\mathcal{S}$ by

$$
l=\max \{|p| ; p<0,[p, 0] \subset \mathcal{S}\}, \quad d=\max \left\{r>0 ; \mathcal{S}_{\mathrm{SDE}, s} \subset \mathcal{S}\right\} .
$$

Clearly, $d \leq l$, and for mean-square stability, it is the parameter $d$ which has to be optimized. For the S-ROCK methods, as can be seen in Figure $4, l$ and $d$ depend on the stage number $m$ and the value of the damping parameter $\eta$. We thus denote these parameters by $l_{m}(\eta)$ and $d_{m}(\eta)$. The following lemmas give important information on the value of $l_{m}(\eta)$ and a bound of the possible values for $d_{m}(\eta)$, the parameter which characterizes the stability domains of our methods.

Lemma $1([7],[8])$. Let $\eta \geq 0$. For all $m \geq 2$, the $m$-stage numerical method (22) has a mean-square stability region $\mathcal{S}_{m}^{\eta}$ with $l_{m}(\eta) \geq c(\eta) m^{2}$, where $c(\eta)$ depends only on $\eta$.

Lemma $2([7],[8])$. For all $m \geq 2$

$$
l_{m}(\eta) \rightarrow 2 m \text { for } \eta \rightarrow \infty .
$$

In view of the above two lemmas we make the following important observation: for any fixed $\eta$, the stability domain along the $p$ axis increases quadratically (Lemma 1 ), but for a given method, i.e., a fixed $m$, increasing the damping $\eta$ to infinity reduces the quadratic growth along the $p$ axis into a linear growth (Lemma 2). Since $d_{m}(\eta) \leq l_{m}(\eta)$ there is no computational saving compared to classical explicit methods for this limit case.

Optimized methods. Our goal is now for a given method to find the value of $\eta$, denoted $\eta^{*}$ which maximize $d_{m}(\eta)$, i.e.,

$$
\eta^{*}=\operatorname{argmax}\left\{d_{m}(\eta) ; \eta \in[0, \infty)\right\} .
$$

The corresponding optimal values $d_{m}\left(\eta^{*}\right)$ for $m \leq 200$ have been computed numerically and are reported in Figure 5 for the Itô S-ROCK methods (19) and in Figure 6 for the Stratonovich S-ROCK methods (22). We also report in the same Figures the values of $l_{m}\left(\eta^{*}\right)$ and $\eta^{*}$. We see that for $\eta=\eta^{*}$, $d_{m}\left(\eta^{*}\right) \simeq l_{m}\left(\eta^{*}\right)$. The dashed and the dash-dotted lines in the plots reporting the values of $d_{m}\left(\eta^{*}\right)$, represent a quadratic and a linear slope, respectively. We clearly see that the portion of the true stability domain included in the stability domain of our numerical methods grows super-linearly (close to quadratically) for both the Itô and the Stratonovich S-ROCK methods. Finally we study the efficiency of the methods by reporting the quantity 

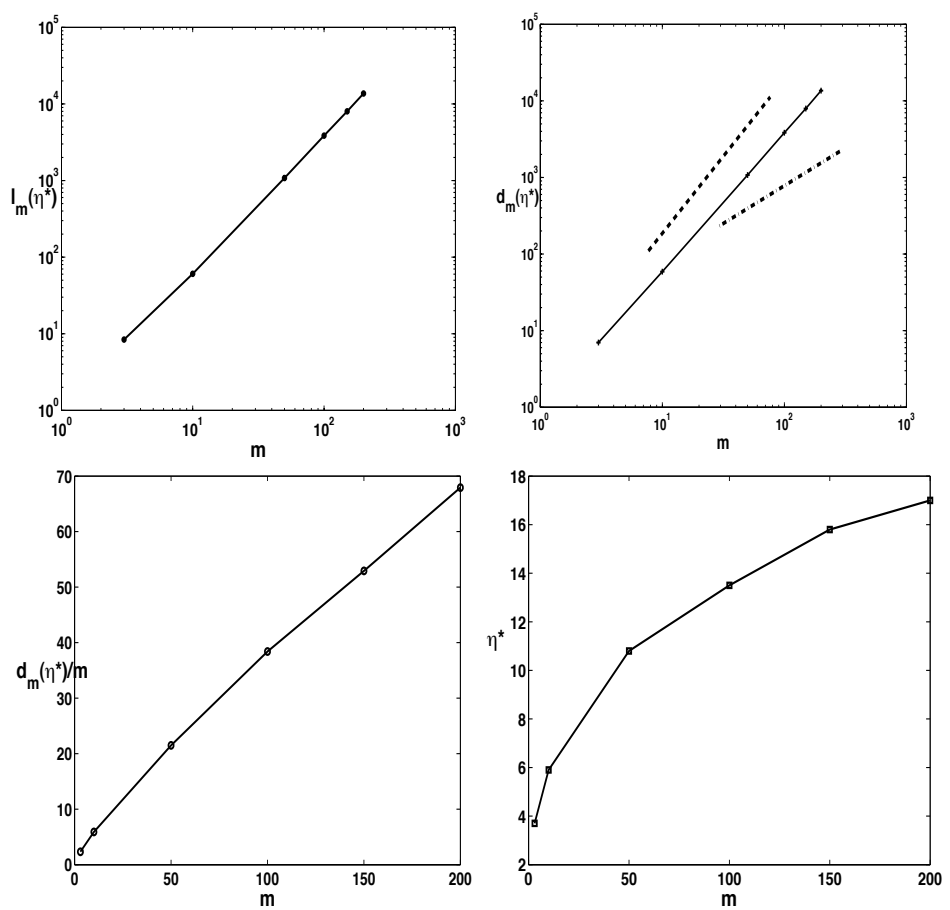

Fig. 5 Values of $\eta^{*}, l^{\eta^{*}}, d^{\eta^{*}}$ as a function of $m$ and the ratio $d_{m}^{\eta^{*}} / m$ (stability versus work) for the Itô S-ROCK methods (19). The dashed and the dash-dotted lines in the upper-right figure represent a quadratic and a linear slope, respectively.

$d_{m}\left(\eta^{*}\right) / m$ (stability versus work). For standard methods this value is small (close to zero for the Euler-Maruyama methods as can be seen in Figure 2 and about $1 / 2$ for the Platen method (see (33) in Section 5)). Another method will be considered in the numerical experiments, namely the RS method [12, p. 187] developed with the aim of improving the mean-square stability of the Platen method. This method has a larger $l$ value than the Platen method but a smaller $d$ value and the efficiency of this method (as measured here) is about 0.3 . We see that S-ROCK methods are orders of magnitude more efficient (for the aforementioned criterion of efficiency related to stability) than standard explicit methods for SDEs.

\section{Numerical illustrations}

In this section we illustrate the efficiency of the S-ROCK methods. As mentioned in the beginning of Section 4, different applications require different 

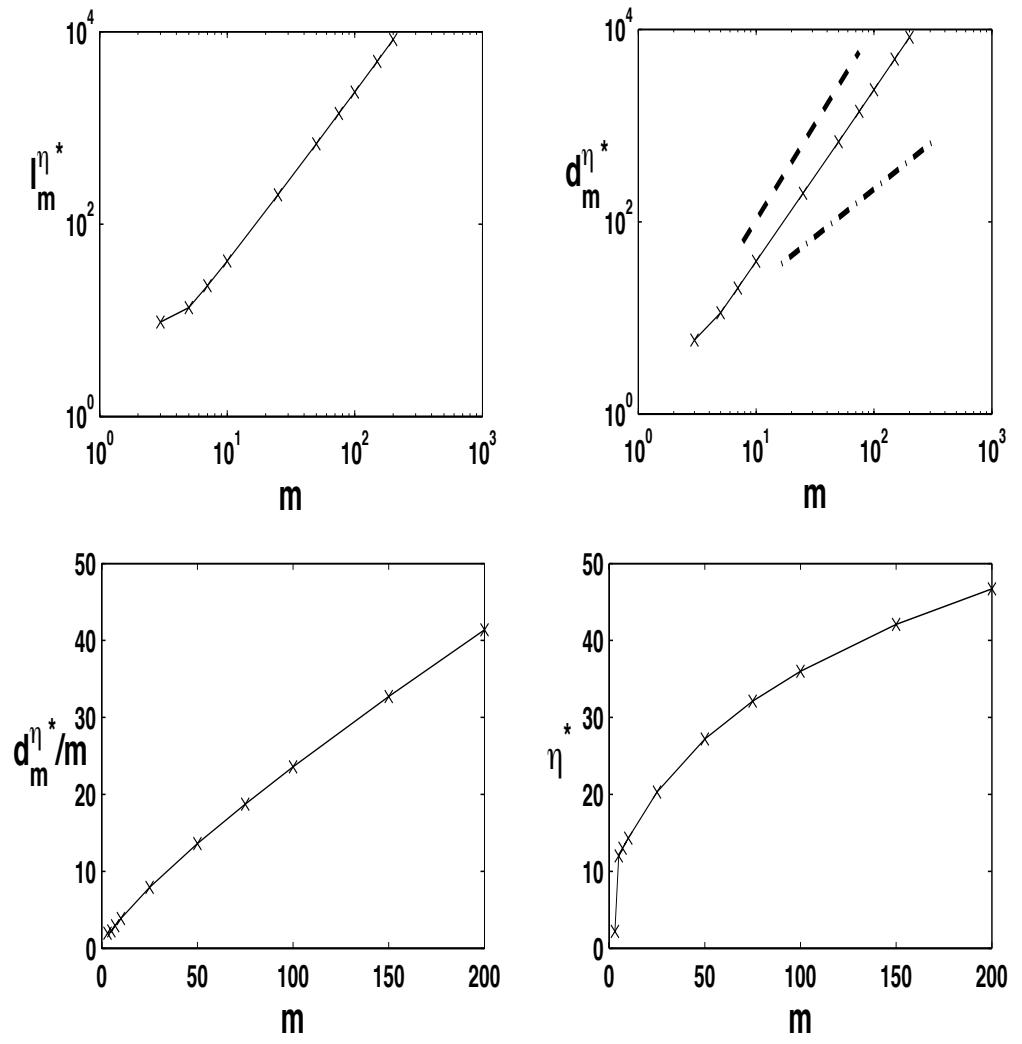

Fig. 6 Values of $\eta^{*}, l^{\eta^{*}}, d^{\eta^{*}}$ as a function of $m$ and the ratio $d_{m}^{\eta^{*}} / m$ (stability versus work) for the Stratonovich S-ROCK methods (22). The dashed and the dash-dotted lines in the upper-right figure represent a quadratic and a linear slope, respectively.

stochastic integrals and we will consider both Itô and Stratonovich SDEs in the following examples. The first example is the heat equation with noise mentioned in the introduction. For this problem we consider the Stratonovich S-ROCK methods. The second example is a chemical reaction modeled by the chemical Langevin equation. The Itô S-ROCK methods will be used for this latter problem. For both examples, we compare the S-ROCK methods with standard explicit methods.

Example 1: heat equation with noise. We consider the SPDE (3), where we choose this time the Stratonovich modeling for the noise. We follow the procedure explained in Section 2 and transform the SPDE in a large system of SDEs 


$$
d Y_{t}^{i}=\frac{Y_{t}^{i+1}-2 Y_{t}^{i}+Y_{t}^{i-1}}{h^{2}}+\mu Y_{t}^{i} \circ d W_{t}, \quad i=1, \ldots, N,
$$

where the symbol $\circ$ denotes the Stratonovich form for the stochastic integral. In our numerical experiments, we compare the Stratonovich S-ROCK methods (22) with two other methods, the method introduced by Platen [35] (denoted PL) given by the two-stage scheme

$$
\begin{aligned}
K_{n} & =Y_{n}+\Delta t f\left(Y_{n}\right)+I_{n} g\left(Y_{n}\right), \\
Y_{n+1} & =Y_{n}+\Delta t f\left(Y_{n}\right)+I_{n} \frac{1}{2}\left(g\left(Y_{n}\right)+g\left(K_{n}\right)\right),
\end{aligned}
$$

and the RS method, introduced by P.M. Burrage [12, p. 187]. This is a 2-stage method constructed with the aim of improving the mean-square stability properties of the Platen method and is given by

$$
\begin{aligned}
K_{n} & =Y_{n}+\frac{4}{9} \Delta t f\left(Y_{n}\right)+\frac{2}{3} J_{n} g\left(Y_{n}\right), \\
Y_{n+1} & =Y_{n}+\frac{\Delta t}{2}\left(f\left(Y_{n}\right)+f\left(K_{n}\right)+\frac{1}{4}\left(g\left(Y_{n}\right)+g\left(K_{n}\right)\right) J_{n} .\right.
\end{aligned}
$$

Both methods have strong order 1 for one-dimensional systems or systems with commutative noise as (4). This is also the case for the Stratonovich S-ROCK methods (22). We have seen, at the end of Section 5, that the stability domains of both methods, PL and RS, cover only a small portion of the stability domain corresponding to the stochastic test equation and this is in contrast with the S-ROCK methods. In Figure 7 we monitor the number of function evaluations (cost $)^{7}$ needed by the various methods to produce stable integrations when increasing the value of $N$, i.e., the stiffness of the problem. For the S-ROCK methods we vary the number of stages to meet the stability requirement (this value is indicated in Figure 7).

We see that the S-ROCK method reduces the computational cost by several orders of magnitude as the stiffness increases. In the same Figure we see the value of the stepsize needed for the different methods, again as a function of $N$. As expected, the standard explicit methods, as PL or RS face severe stepsize restriction as the stiffness increases. This example demonstrates that for classes of SPDEs there is a real advantage in using explicit stabilized methods such as S-ROCK methods. We notice that the stepsize is reduced for the highest value of $N$ for the S-ROCK methods (see Figure 7 (right)). We could have kept the same stepsize but the stage number would then have become quite large. It is well-known for Chebyshev methods that in order to control the internal stability of the method one should avoid computation with a very high stage number [3]. Here we fixed the highest stage number at $m=320$.

${ }^{7}$ By number of function evaluations we mean here the total number of drift and diffusion evaluations. 

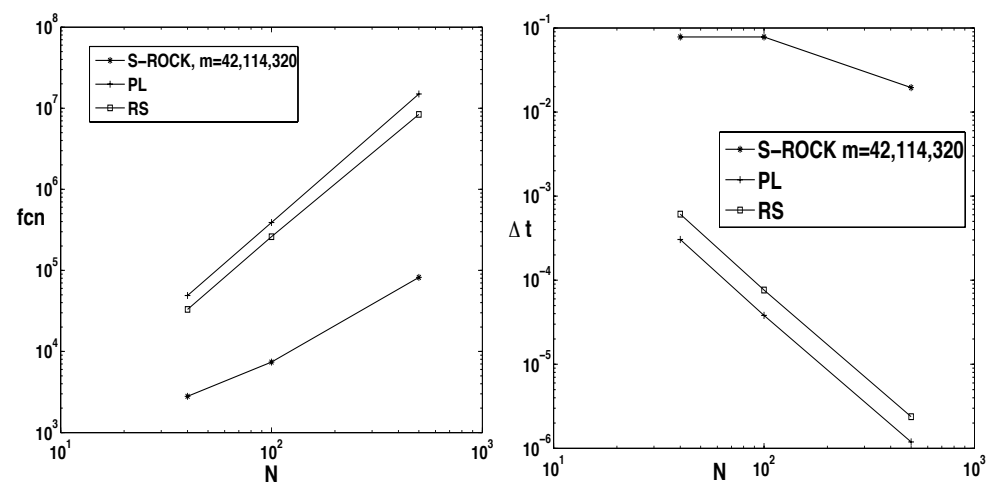

Fig. 7 Function evaluations and stepsize as a function of $N$. For PL and RS, we choose the largest stepsize to have a stable integration of (32) (strong error $<10^{-1}$ ). For the S-ROCK methods, we can vary the stage number $m$ to meet the stability requirement (we fixed the highest stage number at $m=320$ ).

Example 2: a chemical reaction. We know illustrate the use of the Itô SROCK methods. Following [8] we consider a stiff system of chemical reactions given by the Chemical Langevin Equation (CLE). We study the MichaelisMenten system, describing the kinetics of many enzymes. This system has been studied in [24] with various stochastic simulation techniques. The reactions involve four species: $S_{1}$ (a substrate), $S_{2}$ (an enzyme), $S_{3}$ (an enzyme substrate complex), and $S_{4}$ (a product) and can be described as follows: the enzyme binds to the substrate to form an enzyme-substrate complex which is then transformed into the product, i.e.,

$$
\begin{aligned}
S_{1}+S_{2} & \stackrel{c_{1}}{\longrightarrow} S_{3} \\
S_{3} & \stackrel{c_{2}}{\longrightarrow} S_{1}+S_{2} \\
S_{3} & \stackrel{c_{3}}{\longrightarrow} S_{2}+S_{4} .
\end{aligned}
$$

The mathematical description of this kinetic process can be found in [26]. For the simulation of this set of reactions we use the CLE model

$$
d Y(t)=\sum_{j=1}^{3} \nu_{j} a_{j}(Y(t)) d t+\sum_{j=1}^{3} \nu_{j} \sqrt{a_{j}(Y(t))} d W_{j}(t),
$$

where $Y(t)$ is a 4 dimensional vector describing the state of each species $S_{1}, \ldots, S_{4}$. The Itô form used in (38). The functions $a_{j}(Y(t))$, called the propensity functions, give the number of possible combinations of molecules involved in each reaction $j$. For the above system they are given by

$$
a_{1}(Y(t))=c_{1} Y_{1} Y_{2}, a_{2}(Y(t))=c_{2} Y_{3}, a_{3}(Y(t))=c_{3} Y_{3} .
$$


The vectors $\nu_{j}$, called the state-change vectors, describe the change in the number of molecules in the system when a reaction fires. They are given for the three reactions of the above system by $\nu_{1}=(-1,-1,1,0)^{T}, \nu_{2}=$ $(1,1,-1,0)^{T}, \nu_{3}=(0,1,-1,1)^{T}$. We set the initial amount of species as (the parameters are borrowed from [41, Section 7.3])

$$
Y_{1}(0)=\left[5 \times 10^{-7} n_{A} v o l\right], Y_{2}(0)=\left[5 \times 10^{-7} n_{A} v o l\right], Y_{3}(0)=0, Y_{4}(0)=0,
$$

where [.] denotes the rounding to the next integer and $n_{A}=6.023 \times 10^{23}$ is the Avagadro's constant (number of molecules per mole) and vol is the volume of the system.

In the following numerical experiments, we solve numerically the SDE (38) with the Itô S-ROCK methods and the Euler-Maruyama method (6). This latter method is often used for solving the CLE. As the CLE has multidimensional Wiener processes, we use the S-ROCK methods (19). We first compare the solutions along time for the two methods $(t \in[0,50])$, with parameters leading to a non-stiff system for (38). As expected, we observe in Figure 8 a very similar behavior of the two methods.
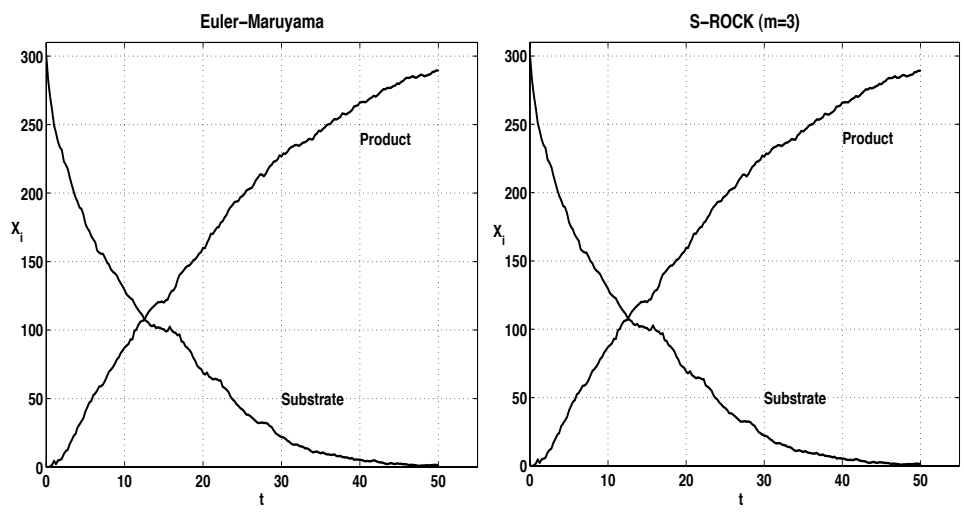

Fig. 8 One trajectory of the Michaelis-Menten system solved with the Euler-Maruyama method (left figure) and the S-ROCK method (right figure) for $c_{1}=1.66 \times 10^{-3}, c_{2}=$ $10^{-4}, c_{3}=0.10$ (the stepsize is $\Delta t=0.25$ and the same Brownian path is used for both methods; $m=3$ for the S-ROCK method).

We next increase the rate of the third reaction in (35)-(37), $c_{3}=10^{2}, 10^{3}, 10^{4}$ corresponding to an increasingly fast production. The resulting CLE becomes stiff and the Euler-Maruyama method is inefficient. In Figure 9 we report the stepsizes and the number of function evaluations needed for the EulerMaruyama and the S-ROCK methods. The stepsize is chosen as $\Delta t=0.25$ for the S-ROCK methods. For the Euler-Maruyama method we select for each value of $c_{3}$ the largest stepsize which leads to a stable integration. Thus, for the Euler-Maruyama method, stability is achieved by reducing the step- 
size while for the S-ROCK method, it is achieved by increasing the stage number $(m=3,7,28,81)$. Notice that for both methods, one evaluation of " $g(Y) d W(t) "$ is needed per stepsize. Thus, by keeping a fixed stepsize, the number of generated random variables remains constant as the stiffness increases for the S-ROCK methods, while this number increases linearly (proportional to the stepsize reduction) for the Euler-Maruyama method. Taking
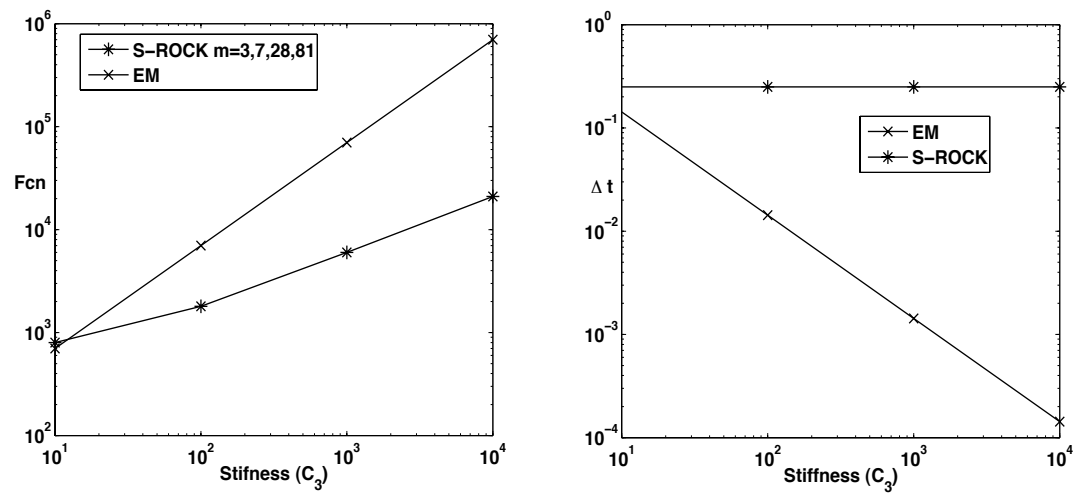

Fig. 9 Numerical solution of (38) with the Euler-Maruyama and the S-ROCK methods. Number of function evaluations as a function of $c_{3}$ for both methods (left figure). Size of the timestep $\Delta t$ as a function of $c_{3}$ (Euler-Maruyama); $\Delta t=0.25$ for the S-ROCK method and the stage number $m$ is adapted to the stiffness (right figure).

advantage of the quadratic growth of the stability domains, we see that the number of function evaluations is reduced by several orders of magnitude when using the S-ROCK methods instead of the Euler-Maruyama method.

\section{References}

1. A.Abdulle, On roots and error constant of optimal stability polynomials, BIT 40 (2000), no. 1, 177-182.

2. A. Abdulle and A.A. Medovikov, Second order Chebyshev methods based on orthogonal polynomials, Numer. Math., 90 (2001), no. 1, 1-18.

3. A. Abdulle, Fourth order Chebyshev methods with recurrence relation, SIAM J. Sci. Comput., 23 (2002), no. 6, 2041-2054.

4. A. Abdulle and S. Attinger, Homogenization method for transport of DNA particles in heterogeneous arrays, Multiscale Modelling and Simulation, Lect. Notes Comput. Sci. Eng., 39 (2004), 23-33.

5. A. Abdulle Multiscale methods for advection-diffusion problems, Discrete Contin. Dyn. Syst. (2005), suppl., 11-21.

6. A. Abdulle and S. Cirilli, Stabilized methods for stiff stochastic systems, C. R. Acad. Sci. Paris, 345 (2007), no. 10, 593-598.

7. A. Abdulle and S. Cirilli, S-ROCK methods for stiff stochastic problems, SIAM J. Sci. Comput., 30 (2008), no. 2, 997-1014. 
8. A. Abdulle and T. Li, S-ROCK methods for stiff Itô SDEs, Commun. Math. Sci. 6 (2008), no. $4,845-868$.

9. A. Abdulle, Y. Hu and T. Li, Chebyshev methods with discrete noise: the tau-ROCK methods, J. Comput. Math. 28 (2010), no. 2, 195-217

10. L. Arnold, Stochastic differential equation, Theory and Application, Wiley, 1974.

11. E. Buckwar and C. Kelly, Towards a systematic linear stability analysis of numerical methods for systems of stochastic differential equations, SIAM J. Numer. Anal. 48 (2010), no. 1, 298-321.

12. P.M. Burrage, Runge-Kutta methods for stochastic differential equations. $\mathrm{PhD}$ Thesis, University of Queensland, Brisbane, Australia, 1999.

13. K. Burrage and P.M. Burrage, General order conditions for stochastic Runge-Kutta methods for both commuting and non-commuting stochastic ordinary differential equation systems, Eight Conference on the Numerical Treatment of Differential Equations (Alexisbad, 1997), Appl. Numer. Math. 28 (1998), no. 2-4, 161-177.

14. P. L. Chow, Stochastic partial differential equations, Chapman and Hall/CRC, 2007.

15. M. Duarte, M. Massota, S. Descombes, C. Tenaudc, T. Dumont, V. Louvet and F. Laurent, New resolution strategy for multi-scale reaction waves using time operator splitting, space adaptive multiresolution and dedicated high order implicit/explicit time integrators, preprint available at hal.archive ouvertes, 2010.

16. W. E, D. Liu, and E. Vanden-Eijnden, Analysis of multiscale methods for stochastic differential equations, Comm. Pure Appl. Math. 58 (2004), no. 11, 1544-1585.

17. D.T. Gillespie, Stochastic simulation of chemical kinetics, Annu. Rev. Phys. Chem. 58 (2007), 35-55.

18. A. Guillou and B. Lago, Domaine de stabilité associé aux formules d'intégration numérique d'équations différentielles à pas séparés et à pas liés. Recherche de formules à grand rayon de stabilité, in Proceedings of the 1er Congr. Assoc. Fran. Calcul (AFCAL), Grenoble, (1960), 43-56.

19. E. Hairer and G. Wanner, Intégration numérique des équations différentielles raides, Techniques de l'ingénieur AF 653, 2007.

20. E. Hairer and G. Wanner, Solving ordinary differential equations II. Stiff and differential-algebraic problems. 2nd. ed., Springer-Verlag, Berlin, 1996.

21. R.Z. Has'minskiǔ, Stochastic stability of differential equations. Sijthoff \& Noordhoff, Groningen, The Netherlands, 1980.

22. M. Hauth, J. Gross, W. Strasser and G.F. Buess, Soft tissue simulation based on measured data, Lecture Notes in Comput. Sci., 2878 (2003), 262-270.

23. D.J. Higham, Mean-square and asymptotic stability of numerical methods for stochastic ordinary differential equations, SIAM J. Numer Anal., 38 (2000), no. 3, 753-769.

24. D.J. Higham, An algorithmic introduction to numerical simulation of stochastic differential equations, SIAM Review 43 (2001), 525-546.

25. P.J. van der Houwen and B.P. Sommeijer, On the internal stage Runge-Kutta methods for large m-values, Z. Angew. Math. Mech., 60 (1980), 479-485.

26. N.G. van Kampen, Stochastic processes in physics and chemistry, 3rd ed., NorthHolland Personal Library, Elsevier, 2007.

27. A.R. Kinjo and S. Takada, Competition between protein folding and aggregation with molecular chaperones in crowded solutions: insight from mesoscopic simulations, Biophysical journal, 85 (2003), 3521-3531.

28. P.E. Kloeden and E. Platen, Numerical solution of stochastic differential equations, Applications of Mathematics 23, Springer-Verlag, Berlin, 1992.

29. V.I. Lebedev, How to solve stiff systems of differential equations by explicit methods. CRC Pres, Boca Raton, FL, (1994), 45-80.

30. T. Li, A. Abdulle and Weinan E, Effectiveness of implicit methods for stiff stochastic differential equations, Commun. Comput. Phys., 3 (2008), no. 2, 295-307.

31. T. Li, Analysis of explicit tau-leaping schemes for simulating chemically reacting systems, SIAM Multiscale Model. Simul., 6 (2007), no. 2, 417-436. 
32. G. Maruyama, Continuous Markov processes and stochastic equations, Rend. Circ. Mat. Palermo, 4 (1955), 48-90.

33. B. Oksendal, Stochastic differential equations, Sixth edition, Springer-Verlag, Berlin, 2003.

34. E. Platen and N. Bruti-Liberati, Numerical solutions of stochastic differential equations with jumps in finance, Stochastic Modelling and Applied Probability, Vol. 64, Springer-Verlag, Berlin, 2010.

35. E. Platen, Zur zeitdiskreten approximation von Itôprozessen, Diss. B. Imath. Akad. des Wiss. der DDR, Berlin, 1984.

36. W. Rümlin, Numerical treatment of stochastic differential equations, SIAM J. Numer. Math., 19 (1982), no. 3, 604-613.

37. Y. Saitô and T. Mitsui, Stability analysis of numerical schemes for stochastic differential equations, SIAM J. Numer. Anal., 33 (1996), no. 6, 2254-2267.

38. V. Thomee, Galerkin finite element methods for parabolic problems, 2nd ed, Springer Series in Computational Mathematics, Vol. 25, Springer-Verlag, Berlin, 2006.

39. E. Vanden-Eijnden, Numerical techniques for multiscale dynamical system with stochastic effects, Commun. Math. Sci., 1 (2003), no. 2, 385-391.

40. J.B. Walsh, An introduction to stochastic partial differential equations, In: École d'été de Prob. de St-Flour XIV-1984, Lect. Notes in Math. 1180, Springer-Verlag, Berlin, 1986.

41. D.J. Wilkinson, Stochastic modelling for quantitative description of heterogeneous biological systems, Nature Reviews Genetics 10 (2009), 122-133. 